\title{
GLORIA GONZÁLEZ AGUDELO: LOS DERECHOS DE SALUD SEXUAL Y REPRODUCTIVA DE LOS MENORES DE EDAD EN EL MARCO DE LOS DERECHOS FUNDAMENTALES ${ }^{1}$
}

\section{SEXUAL RIGHTS AND SEXUAL AND REPRODUCTIVE HEALTH RIGHTS OF MINORS IN THE CONTEXT OF FUNDAMENTAL RIGHTS}

Resumen: El legislador español de 2015, a través de una labor exhaustiva y sistemática -excepcional en materia de menores-, acometió la tarea de reformar aspectos esenciales que afectan a los derechos sexuales y de salud sexual y reproductiva de los menores de edad, especialmente referidos al consentimiento sexual, el aborto y el consentimiento por representación (art. 9.3 L 41/2002, de autonomía del paciente). Estas modificaciones, junto a las restricciones ya existentes en materia de transexualidad o reproducción asistida, permiten deducir una clara intencionalidad de eliminar o limitar la capacidad del menor en la toma de decisiones relevantes en esta materia.

Palabras clave: El Menor de edad, el consentimiento, el consentimiento por representación, el consentimiento sexual, derechos sexuales, derechos de salud sexual y reproductiva, aborto

\begin{abstract}
The Spanish legislator of 2015, through exhaustive and systematic work - exceptional in the field of minors - carried out the task of reforming essential aspects that affect sexual rights and sexual and reproductive health rights of minors: raising the age of sexual consent as well as changes on abortion affecting teenagers under 16 and 17 years old and in the consent by

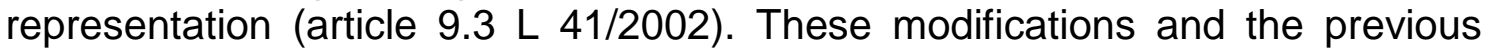
restrictions on transsexuality or assisted reproduction, allow to deduce a clear intention to eliminate or limit the capacity of the minor in the decision making relevant in this matter
\end{abstract}

Keywords: Minor, consent, consent by proxy, sexual rights, sexual and reproductive health rights, abortion

\section{Introducción}

Los indicadores e informes de las Organizaciones Internacionales no cesan de recordarnos la precaria y dramática situación de millones de niñas y niños, adolescentes y jóvenes en el mundo, que no ven reconocido efectivamente su derecho a la salud en general y a la salud sexual y reproductiva en particular, por motivos económicos e infraestructurales en su mayoría, pero también

\footnotetext{
${ }^{1}$ Este trabajo es parte de una investigación más amplia desarrollada en el marco de la subvención PRX 14/00721 para movilidad de profesores e investigadores del Ministerio de Educación, Cultura y Deporte, en el año 2015.
} 
jurídicos e ideológicos, que impiden u obstaculizan una adecuada y oportuna atención, lo que apareja graves consecuencias, como enfermedades de trasmisión sexual, VIH, embarazos no deseados, abortos ilegales, complicaciones gestacionales y en el parto, problemas psicológicos, etc., que en demasiados y prevenibles casos son la causa de miles de muertes (UNICEF, 2016). Si a estos efectos añadimos que son soportadas en su mayor parte por niñas-jóvenes mujeres y que todos aquellos relacionados con el embarazo y/o el aborto son suyos en exclusividad, podemos concluir que las barreras en el ejercicio del derecho a la salud sexual y reproductiva es, en su mayor parte, un problema de género y de discriminación en el acceso al sistema de salud por su condición socioeconómica.

En países desarrollados, como España, en los que el acceso a la sanidad es casi universal -con excepciones- ${ }^{2}$, la atención médica del embarazo y parto de las menores de edad no es discutida, sin embargo, estas no son las únicas cuestiones dudosas en relación con los derechos de salud sexual y reproductiva, pues, incluso este aspecto no está exento de polémica, si se tiene en cuenta que todas las dificultades en el ejercicio de estos derechos tienen que ver con la concepción previa sobre los derechos sexuales y reproductivos y la capacidad de autodeterminación que se reconoce a los menores de edad respecto a ellos.

Un ejemplo de lo dicho lo encontramos en las reformas legislativas de 2015, entre las que destacamos la que eleva la edad del consentimiento sexual a los dieciséis años; limitan la capacidad para decidir el aborto a todas las menores de edad -incluso a las de dieciséis y diecisiete años-; y en los cambios operados en el consentimiento por representación en el ámbito de la salud (art. 9.3 L 41/2002); además del copago y los cambios en el acceso a los métodos anticonceptivos (ONGs, 2016, pp18-20). En tanto la prohibición legal no impide que en la realidad las/los menores sean sexualmente activas, el resultado es que, estos, especialmente las adolescentes, requieren necesariamente servicios de salud sexual y reproductiva, lo que implica una serie de dificultades para ellas y para los terceros relacionados (especialmente los proveedores de servicios de salud), por lo se ven enfrentados a situaciones de hecho complejas, cuya resolución no está casi nunca es sus manos.

Estas no son las únicas dificultades en el ejercicio de los derechos sexuales y reproductivos y de salud sexual y reproductiva (en adelante DSSSR) de los menores de edad, pues, la legislación española ha sido reacia hasta el momento a incluir a los menores de edad en los cambios sociales y jurídicos que se han producido en materia de reproducción asistida y transexualidad, estableciendo la exigencia de la mayoría de edad en ambos casos: en el primer parágrafo $1^{\circ}$ del artículo. 9.5 Ley 41/2002 para hacer uso de las técnicas de reproducción asistida y en una doble vertiente en el caso de la transexualidad, pues se produce en este tema un tratamiento diferenciado en la ley en relación

\footnotetext{
${ }^{2}$ Real Decreto-ley 16/2012, de 20 de abril, de medidas urgentes para garantizar la sostenibilidad del Sistema Nacional de Salud y mejorar la calidad y seguridad de sus prestaciones,.
} 
con su consentimiento. Por una parte, la Ley $3 / 2007$, de 15 de marzo, prevé la rectificación registral de la mención relativa al sexo de las personas, exigiendo la mayoría de edad para poder solicitarla ${ }^{3}$, y por otra, sin que tengan que ser concomitantes o consecuentes, es posible un cambio de sexo físico a través de la operación quirúrgica de reasignación de sexo, estando los menores de edad expresamente exceptuados, según dispone el art. $156 \mathrm{CP}$.

\section{El derecho a la salud sexual y reproductiva del/la menor de edad en el contexto de los derechos sexuales y reproductivos}

Los DSSSR no se encuentran listados taxativamente en las Convenciones sobre derechos fundamentales, aunque parece claro que son parte del contenido de los derechos a la libertad personal y el derecho a la salud, a la autonomía personal y la intimidad y al reconocimiento de la dignidad, esto es, están fundados en un conjunto de derechos y libertades civiles (vida privada; libertad individual; información) y sociales (salud; bienestar) (Saura Estapà, 2015, p.3). Esta afirmación, no está exenta de reticencias y de dificultades, pues, el problema más grave de esta falta de tipicidad es la negación de los mismos y/o de su autonomía por parte de ciertas corrientes ideológicas, lo que puede crear confusión y propiciar diferentes opciones jurídicas en la resolución de los conflictos que necesariamente se presentan.

$\mathrm{Si}$ se hace referencia exclusivamente al derecho a la salud sexual reproductiva, no es difícil concitar un acuerdo casi unánime en torno a la necesidad de cuidados adecuados para la niña-joven mujer embarazada durante el periodo gestacional y el parto -otra cosa es quién debe tomar las decisiones relevantes-. No obstante, el consenso se rompe cuando se reivindica otros derechos de salud sexual y/o reproductiva como la necesidad de ofrecer una información y atención adecuada para el cambio de sexo o el aborto o incluso cuando se plantea la necesidad de ofrecer una información suficiente y el acceso a los métodos anticonceptivos también a los menores de edad (ONGs, 2016, pp. 19).

Gran parte de las dificultades planteadas en el campo de la salud sexual y reproductiva derivan de la falta de concreción sobre los derechos sexuales que están en la base, pues, los derechos de salud sexual y reproductiva son en casi todos los casos, inescindibles de estos. Dada la dificultad de obtener el consenso en esta materia donde las posturas culturales, ideológicas y religiosas se esgrimen como fundamento de la negación, prohibición o de su regulación limitadora, muchas legislaciones (por razones políticas y prácticas), optan por realizar un salto cualitativo argumentando en torno al derecho a la salud, lo que posibilita poner en marcha servicios de salud y regular su uso.

\footnotetext{
${ }^{3}$ El Tribunal Supremo ha presentado una cuestión de inconstitucionalidad en relación al art. 1 de la Ley 3/2007, reguladora de la rectificación registral de la mención relativa al sexo de las personas menores de edad, por presunta vulneración de los artículos 15, 18.1 y 43.1, en relación al 10.1, CE
} 
Se obvia así la discusión en torno a los derechos sexuales que dificultaría el consenso y la aprobación de políticas públicas generales y progresistas más respetuosas con todos los derechos en lisa, buscando garantizar, al menos, la atención obstétrica y aumentar el acceso a la atención calificada y el uso de esta y/o de la misma manera, también la información y disponibilidad de métodos anticonceptivos y profilácticos especialmente frente al SIDA, lo que no deja de ser positivo (UNICEF 2016) pero no garantiza todos los derechos en discusión, incluso el mismo derecho a la salud, en un plano de igualdad.

Ciertamente, cuando se exige el reconocimiento de los DSSSR las cuestiones de fondo no tienen que ver exclusivamente con garantizar el Derecho a la salud. Se está planteando, además, el Derecho a decidir libremente el número de hijos y el esparcimiento; el Derecho a recibir toda la información sobre salud sexual y reproductiva; el Derecho de la mujer a tener control sobre cuestiones relativas a su sexualidad y su cuerpo; el Derecho a la igualdad y no discriminación; y el Derecho a disfrutar de los beneficios del progreso científico, entre otros (Shaw, 2009, p.134; WAS, 2014)

\section{Barreras y limitaciones en el reconocimiento y ejercicio de los derechos de salud sexual y reproductiva del menor de edad}

Los obstáculos e impedimentos en el reconocimiento y ejercicio de DSSSR, afectan especialmente a los grupos más vulnerables como inmigrantes, jóvenes, miembros de minorías étnicas o de una orientación no heterosexual. No debe perderse de vista que este es un problema de género y por tanto la mujer -y si pertenece a uno de estos colectivos con mayor razón-, será quien sufra en mayor medida las consecuencias (ONG, 2016)

Así las cosas y aun cuando en todos los países -incluso en los más desarrollados-, existen obstáculos para ejercer los derechos sexuales y reproductivos, adicionalmente, las/los menores deben enfrentar barreras específicas que no se presentan en el caso de la población adulta (Coram Children's Legal Centre, 2014, pp. 6 y ss.), principalmente referidos a los impedimentos jurídicos y a los condicionamientos culturales o sociales de sus padres o tutores, las creencias religiosas de éstos o sus expectativas en cuanto al momento en que los hijos deben iniciar su actividad sexual (Cook y Dickens, 2000, p.2).

Estas creencias y condicionamientos culturales pueden dificultar el acceso a la información y búsqueda de servicios de salud, pues, sus padres pueden negarles recursos económicos (por ejemplo para el traslado a un centro de asesoramiento o comprar una píldora postcoital), controlar su tiempo y/o el acceso a la información a través de internet, prohibirles explícitamente el uso de los servicios, e impedirles recibir acompañamiento y orientación, etc. (González/Duran, 2010, p.9). También se observa como impedimento relevante la falta de confianza de los propios adolescentes para discutir las cuestiones y problemas relacionadas con la sexualidad y la salud reproductiva, sobre todo si 
se tienen muy asumidos los roles de género, y/o los sentimientos de vergüenza y el temor al juicio -especialmente de los adultos significativos- son muy fuertes (Shaw, 2009, p. 135, Coram Children's Legal Centre, 2014, p. 6; Yarrow et al, 2014, p.152)

El mayor obstáculo es la falta de recursos públicos de salud o la dificultad en el acceso a los mismos, que en España es completamente desigual en función de cada específica Comunidad, y se observa especialmente en el acceso al aborto en la red sanitaria pública (ONGs, 2016, pp 18-20 y 24-27), que se convierten en el caso de los menores, en barreras insalvables, sobre todo, cuando no cuentan con apoyo familiar o la situación económica es precaria. En este ámbito debe hacerse hincapié en las actitudes de los profesionales de la salud, pues son decisivas para fomentar o desestimular el acceso a los servicios de salud sexual y reproductiva, haciéndose imprescindibles los servicios amigables para los jóvenes (Shaw, 2009, p. 135; Cook et al, 2010, p.257), ya que es el criterio decisivo para su uso por los jóvenes.

En esta idea, debe considerarse como barrera específica la propia legislación (Coram Children's Legal Centre, 2014, p. 7) y no solo cuando las prohibiciones son expresas, pues se asocia también a la incertidumbre de los prestadores de servicios de salud respecto a la validez jurídica del consentimiento de sujetos cuya capacidad para decidir y su autonomía es discutida. Esta no es una cuestión baladí, pues, los marcos de actuación profesional a menudo no están definidos con claridad, ni por las leyes ni por los protocolos específicos, sobre todo, en relación con el consentimiento informado, lo cual les genera temor respecto a las medidas que puedan adoptar contra ellos los padres o las autoridades judiciales.

Adicionalmente, debe considerarse las consecuencias sociales y profesionales de ciertas prácticas profesionales en entornos socialmente complejos. En este sentido, por ejemplo, se desarrolla la investigación sistemática de Doran/Nacaroow (2015), en la que se analizan los estudios más importantes publicados entre 1993 y 2014 sobre el aborto, concluyendo que en los países desarrollados aún existen diversas trabas a la hora de interrumpir el embarazo durante las 12 primeras semanas, entre las que destacan: la falta de profesionales capaces o dispuestos a llevar a cabo el procedimiento, el acoso y la estigmatización del personal sanitario que practica los abortos, la falta de información o la falta de recursos para costear el procedimiento.

\section{Los DSSSR del menor de edad y las limitaciones a su autonomía}

Los menores de dieciocho años son titulares de derechos y también deben ser destinatarios de los principios de respeto a la dignidad de las personas y libre desarrollo de la personalidad reconocidos en la Constitución y en los acuerdos y tratados internacionales sobre derechos humanos. Dadas las 
características propias de su desarrollo evolutivo, el ejercicio de los derechos en cada etapa dependerá de múltiples factores concomitantes, bien, de carácter subjetivo, o bien, de carácter objetivo, debiendo proveerse su protección a través de la institución de la tutela, que no es absoluta, pues, solo será admisible para garantizar sus derechos y en la medida en la que el menor no pueda ejercerlos directamente, como sostiene el artículo 5 de la Convención de Derechos del Niño al reconocer las "capacidades evolutivas", por lo que debe ser sustituida progresivamente por una mayor autonomía del menor (STS 141/2000, ATS 10 de marzo de 2016), en la toma de decisiones relevantes, aunque vayan en contra de las ideas o preferencias de los padres y tutores (ver ampliamente González Agudelo, 2016, pp. 18-23).

A pesar de lo anterior, durante el año 2015 se llevaron a cabo en España distintas modificaciones legislativas de gran trascendencia en los derechos del menor de edad y su capacidad de decisión, especialmente en relación con los derechos sexuales y reproductivos, que tienen un efecto directo en los derechos de salud sexual y reproductiva:

\subsection{La elevación de la edad del consentimiento sexual (art. $183 \mathrm{CP}$ )}

El art 183 CP, modificado en la reforma del Código Penal de 2015, eleva la edad del consentimiento sexual de los trece años -en los que estaba- a los dieciséis años, sin establecer parámetros de lesividad en la conducta, y tampoco una clara tipicidad, pues establece como conducta prohibida la siguiente: "El que realizare actos de carácter sexual con un menor de dieciséis años", esto es, cualquier acto de carácter sexual (¿un baile, un frotamiento, penetración?), con y de un menor de dieciséis años entraría de lleno, en principio, en la tipicidad de la conducta, incluso aun cuando se ha incluido la fórmula del art. 183 quater que la autoriza cuando se constate "el consentimiento libre", al que se califica adicionalmente en función de la "proximidad" -concepto totalmente indeterminado- por edad y madurez de los sujetos implicados.

Al no fijarse una edad mínima de diferencia entre víctima y victimario (age span) como presupuesto de la tipicidad, y como la madurez no tiene referentes legales, en realidad se está prohibiendo el ejercicio de la sexualidad, más aun, cuando no se exige abuso, coerción o engaño, para configurar la conducta típica -como en el caso español (Couso, 2009, p. 9-11), pues, siempre será dudoso el comportamiento y será el juez, a posteriori, quien determine sobre la licitud de la conducta.

El resultado de esta argumentación es una aporía, puesto que no puede afirmarse tajantemente que todo contacto sexual de un menor de dieciséis años esté prohibido por la legislación española pero tampoco lo contrario. De aquí se deriva que basta un interés personal (padres, exparejas, fanáticos religiosos, organizaciones conservadores, vecinos, etc.) o las dudas razonables -o motivos ideológicos- en quienes conocen la situación en razón de su cargo (profesores, trabajadores sociales o proveedores de servicios de salud) para 
poner en marcha todo el aparato judicial con las graves consecuencias estigmatizadoras negativas subsiguientes para los implicados y para el propio sistema jurídico a quien se quiere convertir en guardián de la moral y las buenas costumbres.

Las consecuencias de esta decisión van más allá de limitar la capacidad de autodeterminación del menor en cuanto a su libertad sexual, pues, al negársele la capacidad para decidir respecto a los primeros pareciera que por extensión -y directamente en el aborto-, se le incapacita también para tomar decisiones relevantes en el ámbito de la salud sexual y reproductiva (cuidado prenatal, pruebas diagnósticas, aborto terapéutico, cirugía fetal, parto, cesárea, etc.) Introduciéndonos en una falacia circular en la argumentación.

\subsubsection{Anticoncepción y prevención y tratamiento de las ETS}

Las dudas sobre la licitud de la interacción sexual de los jóvenes menores de 16 años se trasladan también al tema de la información y el acceso a los recursos de contra-concepción y profilaxis sexual, fundamentalmente, porque debe tomarse como punto de partida para ofrecer el servicio un hecho de ambigua licitud, que en principio y por defecto está prohibido a todos los menores de dieciséis años, generando dudas, incertidumbres y errores en la atención de los profesionales encargados:

a) Información: Si un menor de cualquier edad solicita información sobre estos temas en un centro del sistema sanitario público o privado de salud, nos encontramos, aparentemente, frente a un conflicto de intereses: por una parte, el derecho a la educación y a la información del menor y, por otra, la prohibición del art. $183 \mathrm{CP}$ en materia sexual relativa a los menores de dieciséis años. Este conflicto tiene fácil resolución en la medida que es imposible saber previamente si ese menor solicita información para llevar a cabo la conducta prohibida o solo trata de obtener un conocimiento adecuado para cuando pueda ejercer sus derechos libremente, por lo que debe primar el derecho a la información (art. $10 \mathrm{CEDH}$, art. $18 \mathrm{CE}$. Ver, caso Gillick vs. el departamento de salud de West Norfolk y Wisbech (3 All ER 402 HL).

b) Acceso a los recursos: Es aquí dónde pueden presentarse los problemas más preocupantes, dada la gravedad de las consecuencias de la obstaculización del acceso a los recursos de contra-concepción y profilaxis sexual de los jóvenes. Es lógico suponer que el profesional debe conocer suficientemente las circunstancias y personalidad de sus pacientes y sus parejas para satisfacer los requerimientos médicos, éticos y legales de la determinación del tratamiento que proponen, si se pretende recomendar y ofrecer el método anticonceptivo y profiláctico más apropiado en el caso concreto (Cook/Cusack/Dickens, 2010, pp.257-258).

En estos casos, si se atiende a la prohibición general de contacto sexual de cualquier menor de dieciséis años contenida en el art. $183 \mathrm{CP}$, podría 
concluirse que ofrecer y especialmente realizar actuaciones sobre el menor para lograr la adecuación de un determinado método anticonceptivo (inyectar, implantar, entregar, etc.) podría llegar a entenderse como una forma de colaboración en el delito, salvo cuando el profesional concluya que efectivamente existe proximidad por edad y madurez, como exige el art. 183 quátur y no se trata de un acto delictivo del que tiene conocimiento por razón de su cargo. Esta decisión obviamente genera un gravamen de incertidumbre, no solo para el profesional, que debe soportar la posibilidad del recurso penal por parte de los tutores o el fiscal, como para los propios menores que pueden ver rechazadas sus pretensiones en función de valoraciones morales 0 ideológicas de quien es el prestador de los servicios.

Esta conclusión es inaceptable desde el punto de vista del derecho a la salud sexual y reproductiva del menor de edad, razón por la cual habrá que acordar que priman estos derechos frente a otros hipotéticos intereses en conflicto, atendiendo el interés superior del menor, que debe entenderse aquí en el sentido de garantizar su actual derecho a la salud e integridad física y psíquica, derecho del que son titulares según el art. $15 \mathrm{CE}$. Existe en este caso un ejercicio claro de un derecho a obtener servicios de salud sexual y reproductiva en el sentido expresado por el art. $20.7 \mathrm{CP}$, por tanto, los proveedores de esos servicios también estarán exentos de responsabilidad criminal (ONU, 2013).

Dado el grado de incertidumbre en la materia y el espaldarazo dado por la STC 145/2015, de 25 de junio de 2015, a la objeción de conciencia en este campo, deberá garantizarse el ejercicio de los DSSSR y por esto se hace necesario protocolizar la actuación de los proveedores de salud sexual y reproductiva, -incluyendo a los farmacéuticos- en los diferentes niveles, garantizando en todos los casos el acceso a la información y los servicios de salud solicitados, aun en aquellos supuestos donde se argumente la objeción de conciencia comprendiendo también la regulación del derecho a la privacidad de los menores (Yarrow et al, 2014, Cook et al, 2010, pp. 152-153 y 182-187; ONU, 2013, par. 31).

c) Píldora poscoital: A pesar de cierta polémica, que incluye la objeción de conciencia alegada por algunos proveedores de servicios de salud sexual y reproductiva, avalada por el Tribunal Constitucional en la Sentencia 145/2015, de 25 de junio de 2015, ha de entenderse que la píldora postcoital o del día después no es abortiva (no debe confundirse con la píldora abortiva RU 486), según indican las organizaciones médicas, pues: "...no puede impedir la implantación de un huevo fecundado" "4, solo obstaculiza la fecundación.

\footnotetext{
${ }^{4}$ Informe de la Sociedad Española de Obstetricia y Ginecología, "Información científica relevante sobre la anticoncepción de urgencia" que remite a la documentación que aprobó la Sociedad Española de Contracepción (SEC), disponible en: http://sec.es/descargas/INFORME_SEGO.pdf, consultada el 23/10/2016
} 


\subsection{Límites del consentimiento informado y consentimiento por representación en materia de salud sexual del menor de edad}

Las modificaciones operadas después de la última reforma de 2015 en los arts. 4, 8 y 9 de la Ley 41/2002, de 14 de noviembre, que contemplan la información asistencial, el consentimiento informado y el consentimiento por representación, tiene gran trascendencia en materia de salud sexual y reproductiva de los menores, especialmente de las mujeres, y no solo porque la Ley Orgánica 11/2015, de 21 de septiembre, modificó directamente lo relativo al consentimiento de las mujeres de dieciséis y diecisiete años para abortar, puesto que dichas modificaciones tienen repercusiones en todos las actuaciones médicas, incluidas aquellas que tienen origen en el ejercicio de la sexualidad y/o la reproducción.

El nuevo art. 9.3, c), de la Ley 41/2002 modifica el sentido de la autonomía del menor, pues, prohíbe el consentimiento por representación de menores emancipados o mayores de dieciséis años, si no tienen la capacidad modificada judicialmente y es capaz intelectual y emocionalmente de comprender el alcance de la intervención, pero añade que en estos casos: "cuando se trate de una actuación de grave riesgo para la vida o salud del menor, según el criterio del facultativo, el consentimiento lo prestará el representante legal del menor, una vez oída y tenida en cuenta la opinión del mismo". Además, el inciso 6 del mismo artículo señala que la decisión por representación, en todos los casos, deberá adoptarse atendiendo siempre "al mayor beneficio para la vida o salud del paciente" y será el médico quien realice la valoración de si la decisión del representante legal es contraria a tal interés.

Para garantizar el derecho a la salud del menor y la autonomía del menor en la toma de decisiones relevantes, el papel de la comunidad médica será de gran trascendencia, pues, deberán establecerse criterios claros que permitan dilucidar cuando se esté frente a grave riesgo para la vida o salud del menor, y no solo de un riesgo hipotético presente en la mayoría de las intervenciones médicas, lo mismo que el esclarecimiento del indefinido y controvertido "mayor beneficio para la vida o salud del paciente". En todos los demás casos, aunque choquen con las preferencias ideológicas o morales de los padres o médicos, deberá atenderse a la voluntad del menor de dieciséis años o más, y de los de menos de dieciséis años con la suficiente madurez, valorada de conformidad con criterios objetivos relacionados con el propio menor y no subjetivos de los adultos implicados.

4.2.2. Consentimiento de los representantes legales de las jóvenes de 16 y 17 años para abortar (art. 9.5, par. 2)

El nuevo art. 9.5 de la Ley 41/2002 de autonomía del paciente, modificado por LO 11/2015, de 21 de septiembre, además del consentimiento de las menores de dieciséis y diecisiete años, introduce la exigencia del 
consentimiento de los representantes legales de estas para abortar, remitiendo al Código Civil para resolver el conflicto que pueda presentarse entre ellos.

No existen razones objetivas basadas en el interés del menor que justifiquen la decisión de limitación de su derecho a la autonomía personal a decidir. En contra de lo argumentado, el cambio normativo aparejará graves consecuencias para las menores que, precisamente, por razones objetivas no comunican a sus padres o tutores su decisión, la mayoría de las veces porque existe desamparo y/o violencia familiar (ACAI, 2014), condenándolas a un aborto clandestino con grave riesgo para su vida o salud o a una maternidad no deseada, agravándose la situación si no se ofrece una oportuna y correcta información ${ }^{5}$.

El Grupo de Expertos sobre Discriminación contra la Mujer en su visita a España en 2014 manifestó que la actual regulación de la objeción de conciencia obstaculiza el acceso de las mujeres al aborto y la nueva regulación del aborto exigiendo el consentimiento de los padres restringe aún más el acceso de las niñas a un aborto seguro y legal, y poner la carga de la prueba en las niñas respecto a que la notificación a sus familiares supone un riesgo. Consideraciones similares se aplican en relación con las niñas menores de dieciséis años (ONU, 2014).

Por otra parte, la jurisprudencia del Tribunal Europeo de Derechos Humanos ya se ha pronunciado en distintas oportunidades sobre la existencia de un derecho en el marco del Convenio a proveer información de manera abierta sobre cuestiones relativas a la interrupción voluntaria del embarazo con base en el art. 10 del Convenio Europeo de Derechos Humanos y, específicamente, sobre el derechos de la mujer embarazada a acceder a información "exhaustiva, completa, necesaria y neutra" sobre la salud del feto y sobre su propia salud "para la toma de una decisión informada" para poder ejercer con eficacia los derechos del art. 8 (caso P. y S. c. Polonia (2013) (Saura Estapà, 2015, pp. 24-26, ONG, 2016, p.25).

\section{Bibliografía}

COOK, Rebeca, CUSACK, Simone y DICKENS, Bernard (2010), Unethical Female Stereotyping in Reproductive Health, International Journal of Gynecology and Obstetrics, núm. 109.

Coram Children's Legal Centre (2014). Qualitative research on legal barriers to young people's access to sexual and reproductive health services: Inception report, London: IPPF. Disponible:

\footnotetext{
${ }^{5}$ El estudio realizado por la Asociación ACAI (Asociación de Clínicas Acreditadas para la Interrupción del Embarazo), señala que el 87\% de mujeres de dieciséis y diecisiete años que abortaron en 2014 si informaron a sus padres o tutores, esto es, casi una de cada 10.
} 
http://www.childrenslegalcentre.com/index.php?page=international_research_pr ojects, consultado el 23/09/2016

DORAN, Frances y NANCARROW, Susan (2015), Barriers and facilitators of access to first-trimester abortion services for women in the developed world: a systematic review, Journal of Family Planning and Reproductive Health Care, № 41; consultado, 18/10/ 2015

GONZÁLEZ, Ana y DURÁN, Juanita, (2010), "Consentimiento informado: capacidad de los menores de tomar decisiones sobre su salud sexual y reproductiva", en IPPF, Derechos sexuales y reproductivos de la gente joven: Autonomía en la toma de decisiones y acceso a servicios confidenciales, IPPF/Profamilia, https://www.ippfwhr.org/sites/default/files/libro_espanol.pdf, disponible:

GONZÁLEZ AGUDELO, Gloria, (2016) "Los derechos sexuales y de salud sexual y reproductiva de los menores de edad y la validez de su consentimiento después de las últimas modificaciones legislativas", Revista Derecho y Salud, Vol 26, № 1

ONU (2014), Working Group on the issue of discrimination against women in law and in practice finalizes country mission to Spain. Disponible en http://www.ohchr.org/EN/NewsEvents/Pages/DisplayNews.aspx?NewsID=1544 4\&LangID=

ONGs (España), (2016), Informe Deficiencias e Inequidad en los servicios de salud sexual y reproductiva en España, disponible: https://www.medicosdelmundo.org/index.php/mod.documentos/mem.descargar/ fichero.documentos_2016_Noviembre_SSR_8d1a1563\%232E\%23pdf

SAURA ESTAPÀ, Jaume, (2015), "El estándar jurídico internacional sobre la interrupción voluntaria del embarazo: reflexiones en perspectiva de derechos humanos", Revista electrónica de estudios internacionales (REEI), ํㅡㄴ 29, 2015 SHAW, Dorothy (2009), Access to sexual and reproductive health for young people: Bridging the disconnect between rights and reality, International Journal of Gynecology and Obstetrics 106

UNICEF (2016), Informe Estado Mundial de la infancia 2016. Una oportunidad para cada niño, disponible, https://old.unicef.es/sites/www.unicef.es/files/estado_mundial_de_la_infancia_2 016.pdf, consultado el 10/10/2016

YARROW, Elisabeth et al (2014), Can a restrictive law serve a protective purpose? The impact of age-restrictive laws on young people's access to sexual and reproductive health services, Reproductive Health Matters, Vol. 22(44) 